\title{
Zur Frage der Insulin=Zuckerbehandlung der Herzkrankheit mit besonderer Beriicksichtigung des Glykogen= und Fettbestandes des Herzmuskels.
}

\author{
Von \\ Mikio Kimura. \\ (木村三樹男) \\ (Aus der Medizinischen Klinik von Prof. Dr. S. Yamakawa, \\ Tohoku-Universität zu Sendai.)
}

Einleitung.

Über den Erfolg der intravenösen Traubenzuckertherapie von Büd ing e ${ }^{1)}$ sind die Autoren der Folgezeit darin einig, dass man dadurch bei Herz- und Gefässerkrankungen nicht selten eine Besserung erzielen kann, wenn auch deren Wirkungsmechanismus noch nicht klar auf der Hand liegt.

Büdingen suchte seinerzeit die Indikation der Zuckerbehandlung in der gestörten Herzfunktion infolge des verminderten Blutzukkergehaltes, indem er diesen Zustand als „, hypoglykämische Cardiodystrophie " bezeichnete, wobei er auch wirklich nicht selten einen niedrigen Blutzuckerwert wie $0,07 \%$ fand. Eine Hypoglykämie, wie Büdingen behauptet, kann man aber bei chronischen Herzinsuffizienten in der Regel nicht bestätigen, also fragt es sich, ob die Zukkerinfusion in diesen Fällen überhaupt eine spezifische Wirkung im Sinne von Büdingen ausübt, oder nur eine unspezifische Reaktion verursacht, welch letztere bei vielen anderen Indikationen derselben Behandlungsweise, z. B. bei der sog. Osmotherapie so angenommen wird.

Die Funktion des Herzmuskels steht mit dessen Glykogengehalt in inniger Beziehung $\left(\mathrm{Külbs}^{2}\right) \mathrm{u}$. Lew is $\left.\mathrm{s}^{3}\right)$. So lässt sich unschwer vermuten, dass, bei akuter oder chronischer Herzsch wäche der Glykogengehalt sehr wahrscheinlich vermindert sein kann, wie es Büdingen annahm. L a s ch ${ }^{4}$ konnte zeigen, dass der Gesamtkohlehydratgehalt besonders beim dekompensierten Herzen eine wesentliche $\mathrm{Ab}$ - 
nahme erfährt. Ishimori $\mathrm{i}^{5}$ und Valdes ${ }^{6}$ gaben ferner an, dass das sukzessive Absterben der einzelnen Teile des herausgenommenen Herzens der ungleich schnellen Abnahme des Kohlehydratvorrats entspricht. Costa $a^{7)}$ vermisste immer das Glykogen im tierexperimentell erzeugten hypertrophischen Herzmuskel. Nach Pomodor o ${ }^{8}$ enthält der Herzabschnitt, an dem experimentell der Infarkt gesetzt war, erheblich weniger Glykogen als der Gesunde.

Auf der anderen Seite kommt die Verfettung des Herzmuskels, die bekanntlich zur Störung der Organfunktion führt, bei verschiedenen akuten Infektionskrankheiten, bei akuten und chronischen Anämien, bei Vergiftungen und endlich auch bei Herzfehlern mehr oder weniger stark ausgeprägt vor. Es fragt sich dabei, wie der vermehrte Fettgehalt des Herzmuskels auf dessen Glykogenbestand wirkt. Es ist sehr wahrscheinlich, dass eine analoge antagonistische Beziehung zwischen den beiden Substanzen auch beim Herzmuskel vorliegt, die manche Autoren schon bei der Leber bestätigten.

So wic die Dinge liegen, ist der Appell unsrerseits wohl berechtigt, dass die Anregung der Glykogenbildung im Herzmuskel und die möglichste Beseitigung des abgelagerten Fettes aus demselben zur Bekämpfung der Herzschwäche in erster Linie angestrebt werden sollen. Dass intravenöse Zuckerinfusion wie in der Leber auch im normalen Herzmuskel eine Glykogenbildung bewirkt, ist schon durch die histologische Untersuchung von Ishimori $i^{5)}$ festgestellt, so scheint es, dass mandas Bü d ing e n sche Behandlung'sverfahren mit Recht zu diesem Zweck verwenden kann. Es bleibt aber dabei noch offen, wie z. B. das Verhältnis bei erkranktem Herzmuskel liegt, und vor allen Dingen wie der Glykogenbestand eines solchen Herzmuskels auf dessen Fettgehalt reagiert. Diese Fragen sollen in dieser Arbeit experimentell klargestellt werden.

Bei der Zuckerbehandlung der Herzkrankheiten wurde ferner der Insulinzusatz von $\mathrm{Osat}{ }^{9}$ vor kurzem vorgeschlagen. Im AnschIuss daran wurde auch die Insulin-Zuckerbehandlung von französischen und italienischen Autoren wie Loeper, Lemaire und Degos, ${ }^{10}$ ) Kisthinios und Gomez, ${ }^{11)}$ Rimbaud, Balmes, Anselme-Martin, ${ }^{12)} \mathrm{Machior} \mathrm{o}^{13)}$ und Bagnaresi ${ }^{14)}$ aufs wärmste empfohlen.

Gegen diese Autoren sahen aber andere Autoren keinen Vorteil durch den Insulinzusatz. So konnten Bobbio und Massobrio, ${ }^{15}$ Penati, Barone und $\mathrm{Croce}^{16)}$ und Ginkel ${ }^{17)}$ dadurch keine Besserung der Krankheitszustände erzielen. Schäffer, Bucka und Friedländer, ${ }^{18)}$ Eismayer und Quincke, ${ }^{19)}$ Ernstene und Alt- 
schule, ${ }^{20)}$ Lasch und Morawitz ${ }^{21)}$ haben sogar einen mehr oder minder deutlichen Schaden durch den Insulinzusatz beobachtet.

In letzter Zeit kam auch $\mathrm{O}$ tom $\mathrm{o}^{22}$ ) in hiesiger Klinik durch den. Versuch mit isolierten Herzen von Frosch und Kaninchen zu folgendem Schluss, dass der Zuckerzusatz zur Durchströmungsflüssigkeit auf sowohl nicht vorbehandelten als auch mit Chloroform geschädigten Herzen erregend wirkt, wobei aber durch Insulinzusatz kein Unterschied nachzuweisen ist.

Die Versuchsergebnisse mancher Autoren uber den Einfluss des Insulins auf den Glykogengehalt des Herzmuskels sind auch nicht immer übereinstimmend. So beobachteten Mc Cormik und Macleod, ${ }^{23)}$ Valdes und Michelazzi ${ }^{24)}$ eine dadurch verursachte Vermehrung, Hepburn und Latschford, ${ }^{25)} \mathrm{Babkin},{ }^{26)} \mathrm{Ochiai,}{ }^{27)} \mathrm{Arnd} \mathrm{t}^{28}$ und Lawrence und Mc Cance ${ }^{29}$ im Gegensatz zu ihnen keine nachweisbare Veränderung des Glykogengehaltes, während dieser bei Edwards und Page, ${ }^{30}$ ) Nagasue, ${ }^{31)}$ Cruickshank und Shrivastava ${ }^{32)}$ u. a. wiederum umgekehrt vermindert vorgefunden wurde.

Die Aufgabe vorliegender Arbeit ist sodann die Indikation des Insulinzusatzes zur Zuckerbehandlung der Herzkrankheiten nachzuprüfen, indem Glykogen- und Fettgehalt des Herzmuskels nach Verabreichung von Zucker und Insulin bei normalen als auch bei phosphorvergifteten Tieren bestimmt wurden.

\section{Methodik.}

Als Versuchstiere, habe ich ausschliesslich Kaninchen gebraucht. Die Fehlerquelle aus ungleichmässiger Verteilung der zu untersuchenden Substanzen auf die verschiedenen Abschnitte des Organs wurde dadurch ausgeschaltet, dass stets das Myokard der linken Kammer als Untersuchungsmaterial benutzt wurde. Die Tiere wurden immer ohne Narkose behandelt und auch ohne Fesselung. Der Tod erfolgte durch Luftembolie, stets 3 Stunden nach der Zuckerinfusion.

Eine Paralleluntersuchung wurde in der Regel mit 3 Kaninchen vorgenommen. Im Text wurde als Ergebnis stets der Durchschnittswert in Betracht gezogen.

Unter den Versuchsmaterialien wurde die Fettaufschwemmung nach dem in dieser Klinik üblichen Verfahren hergestellt, über dessen Einzelheiten ich auf die Arbeiten von $\mathrm{Nom}$ u ra ${ }^{33)}$ u. a. m. verweisen möchte.

Der Glykogengehalt des Herzmuskels wurde wegen der Kleinheit des Versuchsmaterials nach der Mikromethode von Takahata-K u m $\mathrm{e}^{34)}$ und der Fettgehalt gravimetrisch nach $\mathrm{Kumag}$ a w a-Su to scher ${ }^{35}$ ) Verseifungsmethode bestimmt. Letztere erübrigt sich einer Besprechung, da sie allgemein bekannt ist, dagegen könnte eine kurze Beschreibung der ersteren von Interesse sein. 
0,5 g von genau abgewogenem Herzmuskel aus linker Kammer werden in ein Reagenzglas getan, das vorher mit $1 \mathrm{com} 30 \%$ iger $\mathrm{KOH}$ beschickt war, und im Autoklav bei $120^{\circ} \mathrm{C} 30$ Minuten lang erhitzt. Nach genügendem Erkalten wird das Hydrolysat mit cone $\mathrm{HCl}$ zuerst neutralisiert, dann bis auf die Azidität von ungefähr 10\% weiter versetzt und im siedenden. Wasserbade 40 Minuten lang wieder erhitzt. Das Hydrolysat wird nun mit KOH wiederneutralisiert, durch Zusatz von 1,5 ccm 12\% igerLösung von wolframsaurem Natrium und dem gleichen Volumen von $2 / 3 \mathrm{n} \mathrm{H}_{2} \mathrm{SO}_{4}$ enteiweisst und schliesslich mit destilliertem Wasser bis auf $10 \mathrm{ccm}$ aufgefüllt und filtriert.

Das Reduktionsvermögen wird dann mit aliquoten Teilen von $2 \mathrm{~cm}$ klarem Filtrat, einerseits direkt (A) und auf der anderen Seite nach Zerlegung des Zukkers mit heisser $\mathrm{KOH}(\mathrm{B})$, nach Ferricyanmethode von $\mathrm{Ha}$ g e d o rn-J en se ${ }^{36}$ ) ermittelt. Der Unterschied zwischen A und B stellt die wahre Grösse der von Glykogen abgegebenen Zuckermenge dar.

\section{Versuchsergebnisse.}

I. Gegenseitige Verdrängung des Glykogens und Fettes im Herrmuskel unter verschiedenen Umständen.

Dass ein antagonistisches Verhältnis zwischen Fettanhäufung und Glykogenablagerung im Herzmuskel besteht, ist schon von Wegelin, ${ }^{37)}$ MJodowska $a^{38}$ und Junkersdorf und $\mathrm{Kühn}^{39}$ ausgesprochen worden. Die Versuche dieser Autoren wurden grösstenteils bei mit Phlorrhidzin vergifteten Zuständen oder nach langfristigem Hungern angestellt, wodurch die Funktion der Glykogendepots infolge der Erschöpfung oder aus Vitaminmangel schon mehr oder minder stark gelitten haben kann. Diese Fehlerquelle wurde durch meine Versuchsanordnung vollständig ausgeschlossen.

\section{Fett-und Glykogengehalt des Herzmuskels normaler Kaninchen.}

Nach G ü r b e ${ }^{40)}$ unterliegt der Glykogengehalt des Herzmuskels wie auch bei Leber in den verschiedenen Jahreszeiten einer erhebliehen Schwankung. Der Fẹttgehalt der Organe steht gewöhnlich mit dem des Glykogens im umgekehrten Verhältnis. Deswegen ist es ratsam, im Versuche, der sich auf den Glykogen- und Fettgehalt bezieht, die immer nur in derselben Jahreszeit gewonnenen Ergebnisse miteinander zu vergleichen. Da die vorliegende Arbeit in den wärmeren Monaten (von Ende Mai bis Ende Juli) ausgeführt wurde, so führe ich 
hier zunächst als massgebende Vergleichswerte die Analýsenzahlen der Mitte Juli getöteten unvorbehandelten Kaninchen an.

Danach beträgt der Gehalt des Herzmuskels an Glykogen durchschnittlich 0,62\% und der an Fett 2,98\% (Tabelle 1).

Tabelle 1.

Glykogen- und Fettgehalt des Herzmuskels der normalen Kaninchen.

\begin{tabular}{|c|c|c|c|c|}
\hline $\begin{array}{l}\text { Versuchs- } \\
\text { tier-Nr. }\end{array}$ & $\begin{array}{c}\text { Gewicht } \\
\text { (g) }\end{array}$ & $\begin{array}{l}\text { Gewicht des } \\
\text { Herzens }(g)\end{array}$ & $\begin{array}{l}\text { Glykogen } \\
(\%)\end{array}$ & $\begin{array}{l}\text { Fett } \\
(\%)\end{array}$ \\
\hline $\begin{array}{l}1 \\
2 \\
3\end{array}$ & $\begin{array}{l}1900 \\
1700 \\
1520\end{array}$ & $\begin{array}{l}4,2 \\
4,1 \\
3,5\end{array}$ & $\begin{array}{l}0,476 \\
0,628 \\
0,760\end{array}$ & $\begin{array}{l}2,92 \\
2,88 \\
3,14\end{array}$ \\
\hline \multicolumn{2}{|c|}{ Durchschnitt } & & 0,621 & 2,98 \\
\hline
\end{tabular}

Weiter habe ich noch einen anderen Kontrollversuch vorausgeschickt, in dem Kaninchen pro $\mathrm{kg}$ mit $10 \mathrm{ccm} 0,9 \%$ iger NaCl-lösung intravenös behandelt wurden. Er diente zum Vergleich mit den nachfolgenden Versuchen, bei denen die Tiere gewöhnlich auch eine gleiche Menge von Zuckerlösung bzw. Fettemulsion erhielten. Dabei ergab der durchschnittliche Gly kogengehalt 0,62\% und der des Fettes 3,04\%, wodurch festgestellt wurde, dass diese Bestandteile des Herzmuskels durch Zufuhr einer Meng*e indifferenter Flüssigkeit keine nachweisbare Änderung: erfahren (Tabelle 2).

Tabelle 2.

Glykogen- und Fettgehalt des Herzmuskels nach Infusion physiologischer Kochsalzlösung.

\begin{tabular}{c|c|c|c|c}
\hline $\begin{array}{c}\text { Versuchs- } \\
\text { tier-Nr. }\end{array}$ & $\begin{array}{c}\text { Gewicht } \\
(\mathrm{g})\end{array}$ & $\begin{array}{c}\text { Gewicht des } \\
\text { Herzens }(\mathrm{g})\end{array}$ & $\begin{array}{c}\text { Glykogen } \\
(\%)\end{array}$ & $\begin{array}{c}\text { Fett } \\
(\%)\end{array}$ \\
\hline & 1800 & 4,8 & 0,633 & 3,01 \\
4 & 1650 & 3,8 & 0,606 & 2,94 \\
6 & 1600 & 3,5 & 0,612 & 3,16 \\
Durehschnitt & & & $\mathbf{0 , 6 1 7}$ & $\mathbf{3 , 0 4}$
\end{tabular}

\section{Glyjkogen-und Fettbestand des Herzmuskels nach Zuckerinfusion.}

Intravenös infundierter Zucker bewirkt eine Ablagerung des Glykogens in der Leber (Ishimori, Sato, ${ }^{41)}$ Ochiai und Valdes). $\mathrm{Nagao}$ und $\mathrm{Oto} \mathrm{mo}^{42}$ ) stellten in einem derartigen Versuch weiter 
fest, dass bei normalen Tieren Traubenzucker immer eine grössere Ausbeute abgibt als Fruchtzucker, während bei phosphorvergifteten der Fall umgekehrt ist. Ob dieses Ergebnis auch beim Herzmuskel eine Analogie finden kann, habe ich in dieser Arbeit untersucht. Dass intravenöse Infusion von Glukose, Galaktose bzw. Lävulose auch eine Glykogenzunahme des Herzmuskels verursacht, konnte schon Ishimori histologisch nachweisen.

Eine Reihe von wie gewöhnlich gefütterten Kaninchen erhielt pro $\mathrm{kg} 10 \mathrm{ccm} 25 \%$ iger Zuckerlösung in die Vene und wurde 3 Stunden daṇach getötet. Wie man in Tabelle 3 verfolgen kann, vermehrte sich der Glykogengehalt des Herzmuskels nach Zuckergabe ums Doppelte, dagegen war der Fettgehalt um $20 \%$ weniger als bei Kontrolle.

Tabelle 3.

Glykogen- und Fettgehalt des Herzmuskels nach Glukoseinfusion.

\begin{tabular}{|c|c|c|c|c|}
\hline $\begin{array}{l}\text { Versuchs- } \\
\text { tier-Nr. }\end{array}$ & $\begin{array}{l}\text { Gewicht } \\
\text { (g) }\end{array}$ & $\begin{array}{l}\text { Gewicht des } \\
\text { Herzens (g) }\end{array}$ & $\begin{array}{l}\text { Glykogen } \\
(\%)\end{array}$ & $\begin{array}{l}\text { Fett } \\
(\%)\end{array}$ \\
\hline $\begin{array}{l}7 \\
8 \\
9\end{array}$ & $\begin{array}{l}1740 \\
1700 \\
1580\end{array}$ & $\begin{array}{l}3,8 \\
3,5 \\
3,3\end{array}$ & $\begin{array}{l}1,540 \\
1,336 \\
1,472\end{array}$ & $\begin{array}{l}2,52 \\
2,51 \\
2,15\end{array}$ \\
\hline \multicolumn{2}{|c|}{ Durehschnitt } & & 1,449 & 2,39 \\
\hline
\end{tabular}

Aus einem sonst genau so wie oben, aber mit Lävulose angestellten Versuche (Tabelle 4) ergibt sich ferner, dass auch aus dieser Zukkerart die Glykogenneubildung im Herzmuskel auf Kosten des Fettgehaltes gleich wie aus Traubenzucker vor sich geht, wobei aber die Grösse der Glykogenausbeute etwas kleiner, dementsprechend die Reduktion des Fettgehaltes weniger ausgesprochen ist als beim Traubenzuckerversuch. All diese Ergebnisse der Herzmuskelversuche bestätigen die von $\mathrm{Nag} \mathrm{a}^{43)}$ in dieser Klinik bei Leber gemachte Beo-

Tabelle 4.

Glykogen- und Fettgehalt des Herzmuskels nach Lävuloseinfusion.

\begin{tabular}{|c|c|c|c|c|}
\hline $\begin{array}{l}\text { Versuchs- } \\
\text { tier-Nr. }\end{array}$ & $\begin{array}{c}\text { Gewicht } \\
\text { (g) }\end{array}$ & $\begin{array}{l}\text { Gewicht des } \\
\text { Herzens (g) }\end{array}$ & $\begin{array}{c}\text { Glykogen } \\
(\%)\end{array}$ & $\begin{array}{l}\text { Fett } \\
(\%)\end{array}$ \\
\hline $\begin{array}{l}10 \\
11 \\
12\end{array}$ & $\begin{array}{l}2150 \\
2600 \\
2130\end{array}$ & $\begin{array}{l}4,5 \\
5,7 \\
4,7\end{array}$ & $\begin{array}{l}1,296 \\
1,256 \\
1,216\end{array}$ & $\begin{array}{l}3,07 \\
2,20 \\
2,50\end{array}$ \\
\hline \multicolumn{2}{|c|}{ Durchsehnitt } & & 1,256 & 2,59 \\
\hline
\end{tabular}


bachtung, dass bei normalen Tieren Traubenzucker als Glykogenbildner dem Fruchtzucker überlegen ist.

\section{Einfluss der Fettinfusion auf den Glykogenbestand des Herzmuskels der normalen Tiere.}

Wegelin und Mlodowska konnten zeigen, dass nach einer langfristigen und zwangsmässigen Fettfütterung cine beträchtliche Fettablagerung im Herzmuskel entsteht. Um zu prüfen, wie die Fettinfusion den Glykogen- bzw. Fettgehalt des Herzmuskels beeinflusst, habe ich normale Kaninchen mit pro $\mathrm{kg} 10 \mathrm{ccm} 12 \%$ iger Fettemulsion intravenös behandelt und 3 Stunden danach getötet. Der dabei erhobene Glykogenwert war $0,25 \%$, also ungefähr um $60 \%$ kleiner als bei unvorbehandelten Kontrolltieren, dagegen der Fettgehalt, der $3,30 \%$ betrug, war um 10\% höher.

Tabelle 5.

Glykogen- und Fettgehalt des Herzmuskels nach Fettinfusion.

\begin{tabular}{c|c|c|c|c}
\hline $\begin{array}{c}\text { Versuchs- } \\
\text { tier-Nr. }\end{array}$ & $\begin{array}{c}\text { Gewicht } \\
(\mathrm{g})\end{array}$ & $\begin{array}{c}\text { Gewicht des } \\
\text { Herzens }(\mathrm{g})\end{array}$ & $\begin{array}{c}\text { Glykogen } \\
(\%)\end{array}$ & $\begin{array}{c}\text { Fett } \\
(\%)\end{array}$ \\
\hline & & & & \\
13 & 1700 & 3,8 & 0,204 & 3,01 \\
14 & 1700 & 3,7 & 0,244 & 3,60 \\
15 & 1800 & 4,3 & 0,296 & $\mathbf{3 , 3 0}$ \\
\multicolumn{2}{l|}{ Durchschnitt } & & $\mathbf{0 , 2 4 8}$ & $\mathbf{3 , 3 0}$ \\
\end{tabular}

Daraus ergibt sich, dass im Herzmuskel abgelagertes Fett, auch analog wie bei der Leber, das $\nabla$ orratglykogen verdrängt (Tabelle 5).

\section{Kann das nach Infusion im Herzmuskel abgelagerte Fett durch Zuckerinfusion wieder beseitigt werden?}

In diesem Versuch ging man von dem Gesichtspunkt der gegenseitigen Verdrängung des Fettes und Glykogens weiter aus und verfolgte die Frage, ob die Ablagerung infundierten Fettes im Herzmuskel durch Zuckerinfusion aufgehoben werden könne.

Die Kaninchen erhielten pro $\mathrm{kg} 10 \mathrm{ccm} 12 \%$ iger Fettemulsion in die Vene und wurden. 3 Stunden danach wieder pro $\mathrm{kg}$ mit $10 \mathrm{ccm}$ 25\% iger Trauben- resp. Fruchtzuckerlösung intravenös behandelt. Aus den Analysenzahlen der 3 Stunden nach Zucker getöteten Tiere, die in Tabelle 6 und 7 wiedergegeben sind, mit den Ergebnissen der 
Tabelle 6.

Glykogen- und Fettgehalt des Herzmuskels bei nacheinander folgender Fett- und Traubenzuckerinfusion.

\begin{tabular}{c|c|c|c|c}
\hline $\begin{array}{c}\text { Versuchs- } \\
\text { tier-Nr. }\end{array}$ & $\begin{array}{c}\text { Gewicht } \\
(\mathrm{g})\end{array}$ & $\begin{array}{c}\text { Gewicht des } \\
\text { Herzens }(\mathrm{g})\end{array}$ & $\begin{array}{c}\text { Glykogen } \\
(\%)\end{array}$ & $\begin{array}{c}\text { Fett } \\
(\%)\end{array}$ \\
\hline 16 & 1900 & 4,1 & 0,612 & $\mathbf{2 , 5 9}$ \\
17 & 2000 & 4,2 & 0,590 & $\mathbf{2 , 6 8}$ \\
18 & 1880 & $\mathbf{4 , 1}$ & 0,628 & $\mathbf{2 , 2 6}$ \\
Durehschnitt & & & $\mathbf{0 , 6 1 0}$ & $\mathbf{2 , 5 1}$ \\
\hline
\end{tabular}

Tabelle 7.

Derselbe wie oben, aber mit Lävulose angestellte Fersuct.

\begin{tabular}{c|c|c|c|c}
\hline $\begin{array}{c}\text { Versuchs- } \\
\text { tier-Nr. }\end{array}$ & $\begin{array}{c}\text { Gewicht } \\
(\mathrm{g})\end{array}$ & $\begin{array}{c}\text { Gewicht des } \\
\text { Herzens }(\mathrm{g})\end{array}$ & $\begin{array}{c}\text { Glykogen } \\
(\%)\end{array}$ & $\begin{array}{c}\text { Fett } \\
(\%)\end{array}$ \\
\hline & 1850 & 4,0 & 0,571 & 2,56 \\
19 & 1900 & 4,1 & 0,569 & 2,64 \\
20 & 1700 & 3,6 & 0,591 & $\mathbf{2 , 4 7}$ \\
\multicolumn{2}{l|}{ Durehschnitt } & & $\mathbf{0 , 5 7 7}$ & $\mathbf{2 , 5 6}$ \\
\hline
\end{tabular}

schón oben erörterten Versuche verglichen, lässt sich folgendes hervorheben. Im Vergleich zur einfachen Fettinfusion (Tab. 5) wurde der Fettgehalt des Herzmuskels durch Zuckerbehandlung ungefähr um $23 \%$, also über den durch Infusion vermehrten Betrag reduziert, während sich inzwischen der Glykogengehalt ca. ums doppelte vergrösserte. Der Zucker verdrängte dabei nicht nur das nach Infusion abgelagerte Fett, sondern gleichzeitig auch den labilen Anteil des präformierten Fettes aus dem Herzmuskel, wie dies bei einfacher Zukkerinfusion (Tab. 3 und 4) der Fall war.

\section{Glyjkogen-bzw. Fettbestand des Herzmuskels nach gleich- zeitiger Darreichung von Zucker und Fett.}

Wie sich aus den oben angeführten Versuchen erhellt, verursacht die Zuckerinfusion eine Ablagerung des Glykogens im Herzmuskel auf Kosten des Fettgehaltes, während die Fettinfusion einen umgekehrten Erfolg bewirkt. In diesem Versuch wurde weiter untersucht, wie die Erscheinung der gegenseitigen Verdrängung den Glykogenbestand des Herzmuskels ändert bei kombinierter Anwendung von Zucker und Fett. 
In den Versuchen, deren Ergebnisse in Tabelle 8 und 9 wiedergegeben sind, erhielten die Versuch stiere pro $\mathrm{kg} 10 \mathrm{~cm} 12 \%$ iger Fettemulsion, die auf eine Konzentration von $25 \%$ mit Glukose oder Lävulose beschickt worden war, in die Vene. Der Tod der Tiere erfolgte wie üblich 3 Stunden nach der Zuckerdarreichung.

Tabelle 8.

Glykogen- und Fettgehalt des Herzmuskels nach gleichzeitiger Fett- und Traubenzuckerinfusion.

\begin{tabular}{c|c|c|c|c}
\hline $\begin{array}{c}\text { Versuchs- } \\
\text { tier-Nr. }\end{array}$ & $\begin{array}{c}\text { Gewicht } \\
(\mathbf{g})\end{array}$ & $\begin{array}{c}\text { Gewicht des } \\
\text { Herzens }(\mathbf{g})\end{array}$ & $\begin{array}{c}\text { Gigkogen } \\
(\%)\end{array}$ & $\begin{array}{c}\text { Fett } \\
(\%)\end{array}$ \\
\hline & 1800 & 5,2 & 0,499 & 3,00 \\
$\mathbf{2 2}$ & $\mathbf{1 6 0 0}$ & 3,2 & 0,571 & $\mathbf{2 , 7 5}$ \\
$\mathbf{2 4}$ & $\mathbf{1 6 0 0}$ & 3,2 & 0,547 & $\mathbf{2 , 5 1}$ \\
Durchsehnitt & & & $\mathbf{0 , 5 3 9}$ & $\mathbf{2 , 7 5}$
\end{tabular}

Tabelle 9.

Derselbe Versuch wie oben, aber statt Glukiose Lävulose angewendet.

\begin{tabular}{c|c|c|c|c}
\hline $\begin{array}{c}\text { Versuchs- } \\
\text { tier-Nr. }\end{array}$ & $\begin{array}{c}\text { Gewicht } \\
(\mathrm{g})\end{array}$ & $\begin{array}{c}\text { Gewicht des } \\
\text { Herzens }(\mathrm{g})\end{array}$ & $\begin{array}{c}\text { Glykogen } \\
(\%)\end{array}$ & $\begin{array}{c}\text { Fett } \\
(\%)\end{array}$ \\
\hline & 1700 & 4,4 & 0,498 & 2,91 \\
25 & 1500 & 3,1 & 0,535 & 3,13 \\
26 & 1680 & 3,7 & 0,540 & 2,40 \\
Durchschnitt & & & $\mathbf{0 , 5 2 4}$ & $\mathbf{2 , 8 1}$
\end{tabular}

Nach dieser gleichzeitigen Darreichung von Zucker und Fett nimmt der Glykogengehalt des Herzmuskels gerade eine Mittelstellung zwischen den bei resp. mit Zucker und Fett einseitig behandelten Tieren gefundenen Werten. Auch in diesen Versuchen wurde ein bischen mehr Glykogen nach der Traubenzuckerinfusion beobachtet als nach Fruchtzucker.

Die Ergebnisse dieser Versuchsreihe weisen darauf hin, dass parenterale Zuckerdarreichung zur Bereicherung des Glykogens im Hexzmuskel auf Kosten dessen Fettgehaltes führt, und dagegen eine Fettinfusion einen umgekehrten Zustand hervorbringen kann, was alles die schon bei Leber beobachtete Erscheinung von gegenseitiger Verdrängung des Glykogens und Fettes wieder bestätigt. 
II. Wirkung der Zuckerinfusion auf den Glykogen- und Fettbestand des phosphorvergifteten $\mathrm{Herzens.}$

Die vorhergehenden Versuche betrafen ausschliesslich die normalen Tiere. In den folgenden Versuchen will ich weiter die Wirkung: der Zuckerinfusion auf den Glykogen- und Fettbestand des erkrankten Herzmuskels untersuchen. Da Veränderungen des Herzens gleich wie bei der menschlichen Pathologie im Tierversuch schwer nachzuahmen sind, so begnügte ich mich hier nur mit phosphorvergiftetem Herzen zu arbeiten, wie schon auch Rosenfeld, ${ }^{44)} \mathrm{Klewitz-Kirch-}$ heim ${ }^{45}$ u. a.m. in dergleichem Versuch vorgenommen hatten. Die Tiere erhielten dazu $0,5 \mathrm{ccm}$ Süssmandelöl pro kg Körpergewicht, das gelben Phosphor im Verhältnis von $0,5 \%$ enthielt, einmal subkutan. Der Tod erfolgte 48 Stunden nach Phosphorinjektion.

\section{Glykogen-und Fettgehalt des Herzmuskels der. phosphorvergifteten Tiere.}

Durch Phosphorvergiftung findet bekanntlich eine deutliche Verfetteng des Herrmuskels statt, die sich durch beträchtliche Zunahme des Fettes und gleichzeitige Verminderung des Vorratglykogens charakterisiert. So erhöhte sich auch in diesem Vergiftungsversuch der Fettgehalt des Herzmuskels auf $4,24 \%$, der um ca. $42 \%$ grösser als der Normalwert war. Im Gegensatz dazu reduzierte sich der Glykogengehalt auf $0,20 \%$, machte also $43 \%$ des Normalwertes aus (Tabelle 10).

Tabelle 10.

Glykogen- und Fettgehalt des Herzmuskels nach sublutaner Phosphorinjektion.

\begin{tabular}{c|c|c|c|c}
\hline $\begin{array}{c}\text { Versuchs- } \\
\text { tier-Nr. }\end{array}$ & $\begin{array}{c}\text { Gewicht } \\
(\mathbf{g})\end{array}$ & $\begin{array}{c}\text { Gewicht des } \\
\text { Ferzens }(\mathbf{g})\end{array}$ & $\begin{array}{c}\text { Glykogen } \\
(\%)\end{array}$ & $\begin{array}{c}\text { Fett } \\
(\%)\end{array}$ \\
\hline 28 & 2100 & 4,4 & 0,264 & $\mathbf{5 , 6 1}$ \\
29 & 2400 & 4,5 & 0,160 & $\mathbf{3 , 5 1}$ \\
30 & 2400 & $\mathbf{4 , 3}$ & 0,184 & $\mathbf{3 , 6 0}$ \\
\multicolumn{2}{c|}{ Durchsehnitt } & & $\mathbf{0 , 2 0 2}$ & $\mathbf{4 , 2 4}$
\end{tabular}

\section{Zuckerbehandlung der phosphorvergifteten Tiere.}

Es wurde weiter untersucht, wie die Zuckerdarreichung der bei Phosphorvergiftung entstehenden Verfettung des Herzmuskels entge- 
genwirken kann. Die Tiere erhielten nach der Phosphorinjektion 6 mal in Intervallen von 7,5 Stunden eine Zuckermenge von pro $\mathrm{kg} 10$ ccm $25 \%$ iger Lösung in die Vene. Daraufhin wurden sie 3 Stunden nach der letzten Zuckergabe getötet.

Tabelle 11.

Glykogen- und Fettgehalt des Herzmuskels der Phosphorkaninchen bei intravenöser Traubenzuckerbehandlung.

\begin{tabular}{c|c|c|c|c}
\hline $\begin{array}{c}\text { Versuchs: } \\
\text { tier-Nr. }\end{array}$ & $\begin{array}{c}\text { Gewicht } \\
(\mathrm{g})\end{array}$ & $\begin{array}{c}\text { Gewicht des } \\
\text { Herzens }(\mathrm{g})\end{array}$ & $\begin{array}{c}\text { Glykogen } \\
(\%)\end{array}$ & $\begin{array}{c}\text { Fett } \\
(\%)\end{array}$ \\
\hline 31 & 2300 & 5,0 & 0,664 & \\
32 & 2400 & 4,9 & 0,744 & 3,59 \\
33 & 2200 & 4,1 & 0,856 & 3,10 \\
Durehsehnitt & & $\mathbf{0 , 7 5 5}$ & $\mathbf{3 , 3 4}$
\end{tabular}

Tabelle 12.

Derselbe Versuch wie oben, aber mit Lävulose behandelt.

\begin{tabular}{c|c|c|c|c}
\hline $\begin{array}{c}\text { Versuchs- } \\
\text { tier-Nr. }\end{array}$ & $\begin{array}{c}\text { Gewicht } \\
(\mathbf{g})\end{array}$ & $\begin{array}{c}\text { Gewicht des } \\
\text { Herzens }(\mathrm{g})\end{array}$ & $\begin{array}{c}\text { Glykogen } \\
(\%)\end{array}$ & $\begin{array}{c}\text { Fett } \\
(\%)\end{array}$ \\
\hline & & & & \\
34 & 2600 & 6,1 & 0,776 & 2,70 \\
35 & 2300 & 4,6 & 0,872 & 2,38 \\
36 & 2200 & 4,0 & 0,824 & 2,87 \\
\multicolumn{2}{l|}{ Durchsehnitt } & & $\mathbf{0 , 8 2 4}$ & $\mathbf{2 , 6 5}$
\end{tabular}

Der Erfolg der Zuckerdarreichung war schon ziemlich zufriedenstellend (Tabelle 11 u. 12). Der Glykogengehalt des Herzmuskels der vergifteten Tiere nach der Trauben-oder Fruchtzuckeranwendung war durchschnittlich 0,75 bzw. 0,82\%. Diese Werte waren, wenn auch kleiner als die der mit Zucker behandelten, nicht vergifteten Tiere (Tabelle 3 u. 4), doch erheblich grösser als die der ebenso vergifteten, aber nicht behandelten (Tabelle 10) und erreichten den Normalwert in derselben Jahreszeit annähernd. Entsprechend der Glykogenzunahme war der Fettgehalt des vergifteten Herzmuskels nach Traubenzuckerbehandlung auf 3,34\% und nach Fruchtzuckerbehandlung auf $2,65 \%$ abgefallen, wurde also im Vergleich bei den nicht zuckerbehandelten Fällen (Tabelle 8 ) um 21 resp. 14\% reduziert.

Aus den Versuchen ergibt sich, dass dieZuckerinfusion den Glykogengehalt des Hexzmuskels der phosphorvergifteten Tiere auch wie bei den normalen Tieren erhöht, wodurch zugleich eine Abschwächung der für Phosphorvergiftung charakteristischen Fettansammlung er- 
zielt wird. Als Glykogenbildner ist dabei im Gegensatz zu normalen Tieren die Lävulose der Glukose vorzuziehen, was Nagao in dieser Kilinik schon bei der Leber der vergifteten Tiere zeigen konnte.

III. Wirkung des Insulins auf den Glykogenund Fettgehalt des Herzmuskels.

Wie eingangs erörtert, sind die Versuchsergebnisse der vorerwähnten Autoren über den Einfluss des Insulins auf den Glykogenbestand des Herzmuskels nicht immer analog. Ich habe in diesem Experiment untersucht, wie das Insulin für sich allein oder zusammen mit Zucker verabreicht, auf den Glykogen- und Fettbestand des Herzmuskels der normalen, phosphorvergifteten und ausserdem auch noch mit Fettemulsion vor behandelten Tieren wirkt.

\section{Einfluss des Insulins auf Glykogen-und Fettbestand des Herzmuskels der normalen Tiere.}

Insulin (Toronto) wurde in Menge von 0,5 bzw. 1,0 E. H. pro kg: den normalen wie gewöhnlich gefütterten Kaninchen unter die Haut verabreicht, die 3 Stunden danach zur Analyse getötet wurden.

Der Glykogengehalt des Herzmuskels betrug je nach der Grösse der angewendeten Insulinmenge 0,55 resp. 0,48\%, und der an Fett 2,57 resp. 2,61\%. Im Vergleich mit den Normalwerten (Tabelle 1) erkennt man nun, dass durch Insulindarreichung das Glykogen des Herzmuskels der normalen Tiere erheblich vermindert, während das Fett leicht vermehrt wird.

Tabelle 13.

Glykogen- und Fettgehalt des Her:muskels bei einfacher Insulinbehandlung.

\begin{tabular}{|c|c|c|c|c|c|}
\hline $\begin{array}{l}\text { Versuchs- } \\
\text { tier-Nr. }\end{array}$ & $\begin{array}{c}\text { Insulin }(\mathbf{E}) \\
\text { pro } \mathrm{kg}\end{array}$ & $\begin{array}{l}\text { Gewicht } \\
\text { (g) }\end{array}$ & $\begin{array}{l}\text { Gewicht des } \\
\text { Herzens }(\boldsymbol{g})\end{array}$ & $\begin{array}{c}\text { Glykogen } \\
(\%)\end{array}$ & $\begin{array}{l}\text { Fett } \\
(\%)\end{array}$ \\
\hline $\begin{array}{l}37 \\
38 \\
39\end{array}$ & $\begin{array}{l}0,5 \\
0,5 \\
0,5\end{array}$ & $\begin{array}{l}2040 \\
1720 \\
1700\end{array}$ & $\begin{array}{l}4,8 \\
3,9 \\
3,8\end{array}$ & $\begin{array}{l}0,512 \\
0,544 \\
0,588\end{array}$ & $\begin{array}{l}\mathbf{2}, 73 \\
\mathbf{2 , 4 9} \\
\mathbf{2 , 5 1}\end{array}$ \\
\hline \multicolumn{2}{|c|}{ Durchschnitt } & & & 0,548 & 2,57 \\
\hline $\begin{array}{l}40 \\
41 \\
42\end{array}$ & $\begin{array}{l}1,0 \\
1,0 \\
1,0\end{array}$ & $\begin{array}{l}2050 \\
2000 \\
1900\end{array}$ & $\begin{array}{l}4,7 \\
5, \overline{5} \\
4,2\end{array}$ & $\begin{array}{l}0,526 \\
0,424 \\
0,496\end{array}$ & $\begin{array}{l}2,69 \\
2,96 \\
2,20\end{array}$ \\
\hline \multicolumn{2}{|c|}{ Durehschnitt } & & & 0,482 & 2,61 \\
\hline
\end{tabular}


Insulin bewirkt also keine Glykogenablagerung im Herzmuskel, sondern dessen Verbrauch, und zumal in desto grösserer Menge, je mehr Hormon dabei verwendet wird (Tabelle 13).

\section{Glykogen-und Fettgehalt des Herzmuskels nach gleich- zeitiger Darreichung von Insulin und Zueker.}

Die wie gewöhnlich gefütterten Kaninchen erhielten pro $\mathrm{kg}$ 0,5 bzw. 1,0 E. H. Insulin subkutan und dazu noch Glukose bzw. Lävulose in einer Menge von $10 \mathrm{ccm} 25 \%$ iger Lösung pro kg intravenös.

Tabelle 14.

Glykogen- und Fettgehalt des Herzmuskels bei kombinierter Insulin-Zuckerbehandlung.

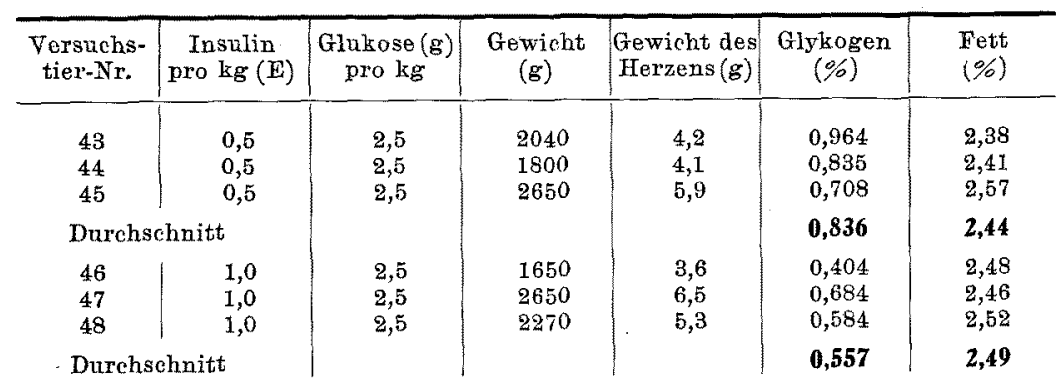

Tabelle 15.

Derselbe Versuch wie oben, aber mit Lävulose behandelt.

\begin{tabular}{|c|c|c|c|c|c|c|}
\hline $\begin{array}{l}\text { Versuchs- } \\
\text { tiel-Nr. }\end{array}$ & $\begin{array}{c}\text { Insulin (E) } \\
\text { pro kg }\end{array}$ & $\begin{array}{c}\text { Lävulose }(g) \\
\text { pro kg }\end{array}$ & $\begin{array}{l}\text { Gewicht } \\
(\mathrm{g})\end{array}$ & $\begin{array}{l}\text { Gowicht des } \\
\text { Herzens }(g)\end{array}$ & $\begin{array}{c}\text { Glykogen } \\
(00)\end{array}$ & $\begin{array}{l}\text { Fett } \\
(\%)\end{array}$ \\
\hline 49 & 0,5 & 2,5 & 2100 & 4,3 & 0,771 & 2,61 \\
\hline 50 & 0,5 & 2,5 & 1850 & 4,0 & 0,718 & 2,53 \\
\hline 51 & 0,5 & 2,5 & 1770 & 4,5 & 0,820 & 2,49 \\
\hline \multicolumn{2}{|c|}{ Durchschnitt } & & & & 0,768 & 2,54 \\
\hline 52 & 1,0 & 2,5 & 2180 & 5,0 & 0,551 & 2,44 \\
\hline 53 & 1,0 & 2,5 & 2300 & 4,9 & 0,587 & 8,60 \\
\hline 54 & 1,0 & 2,5 & 2200 & 4,7 & 0,365 & 2,93 \\
\hline \multicolumn{2}{|c|}{ Durchschnitt } & & & & 0,501 & 2,66 \\
\hline
\end{tabular}

Wie sich in Tabelle 14 und 15 verfolgen lässt, waren die Glykogenwerte des Herzmuskels nach kombinierter Applikation von Zucker und Insulin viel geringer als die bei alleiniger Zuckeranwendung. Dar- 
aus ist zu ersehen, dass das Insulin die durch Zuckerinfusion verursachte Glykogenablagerung im Herzmuskel nicht fördert, sondern im Gegenteil hemmt, und zumal diese Wirkung mit der Grösse der ang'ewandten Insulinmenge immer stärker vor sich geht.

\section{Wirkt Insulin entgegen dem durch Fettinfusion bedingten Glykogenverluste im Herzmuskel?}

Im obigen Versuch wurde festgestellt, dass die Insulinanwendung ungünstig auf den Glykogengehalt des Herzmuskels wirkt, auch wenn dic Glykogenneubildung aus infundiertem Zucker vor sich geht. Wie man aus Tabelle 5 ersieht, wird das Vorratglykogen des Herzmuskels nach Infusion einer Fettaufschwemmung: wegen der gegenseitigen Verdrängung der beiden Substanzen vermindert. S a k a m a k $\mathbf{i}^{46}$ untersuchte in dieser Klinik die Wirkung des Insulins auf derartige aber in der Leber sich abspielende Verdrängungserscheinung und konnte zeigen, dass das Hormon einen durch Fettinfusion verursachten Glykogenverlust nicht unterdrücken kann. Ob die Analogie auch bezüglich des Glykogenbestandes des Herzmuskels besteht, wurde folgender Versuch unternommen.

3 Stunden nach intravenöser Infusion 12\%iger Fettemulsion in einer Menge von $10 \mathrm{ccm}$ pro $\mathrm{kg}$ wurden die Tiere mit 0,5 bzw. 1,0 E. H. Insulin pro $\mathrm{kg}$ subkutan behandelt.

Aus dem Ergebnis dieses Versuchs (Tabelle 16), nach dem das Hearzglykogen ungefähr ein Drittel des Normalwertes darstellte und sogar noch ein bischen weniger als bei einfacher Fettinfusion betrug, kann man leicht verstelien, dass der Insulinzusatz den durch Fettinfu-

Tabelle 16.

Glykogen- und Fettgehalt des Herzmuskels der mit Fettaufschwemmung vorbehandelten Tiere nach Insulinapplikation.

\begin{tabular}{|c|c|c|c|c|c|}
\hline $\begin{array}{l}\text { Versuchs } \\
\text { tier-Nr. }\end{array}$ & $\begin{array}{l}\text { Insulin (E) } \\
\text { pro kg }\end{array}$ & $\begin{array}{l}\text { Gewicht } \\
(\mathrm{g})\end{array}$ & $\begin{array}{c}\text { Gewieht des } \\
\text { Herzens (g) }\end{array}$ & $\begin{array}{c}\text { Glykogen } \\
(\%)\end{array}$ & $\begin{array}{l}\text { Fett } \\
(\%)\end{array}$ \\
\hline 55 & 0,5 & 2110 & 4,3 & 0,218 & 3,30 \\
\hline 56 & 0,5 & 2000 & 4,2 & 0,271 & 3,51 \\
\hline 57 & 0,5 & 2250 & 4,0 & 0,196 & 3,34 \\
\hline \multicolumn{2}{|c|}{ Durehsehnitt } & & & 0,228 & 3,38 \\
\hline 58 & 1,0 & 1800 & 3,7 & 0,203 & 3,57 \\
\hline 59 & 1,0 & 1800 & 4,0 & 0,188 & 3,69 \\
\hline 60 & 1,0 & 1980 & 4,1 & 0,209 & 4,44 \\
\hline \multicolumn{2}{|c|}{ Durehschnitt } & & & 0,200 & 3,83 \\
\hline
\end{tabular}


sion verursachten Glykogenverlust nicht ausgleicht, sondern mehr verstärkt.

Zur Kontrolle wurde auch das umgekehrte Verhältnis untersucht, wie die Verdrängung des nach Fettinfusion im Herzmuskel abgelagerten Fettes durch Zuckerbehandlung bei Anwendung von Insulin beeinflusst wird.

3 Stunden nach Fettinfusion erhielten die Kaninchen pro kg 10 ccm 25\%iger Trauben- sowie Fruchtzuckerlösung intravenös und gleichzeitig auch 0,5 oder 1,0 E.H. Insulin subkuten (Tabelle 17 u. 18).

Tabelle 17.

Die Tiere näh vorausgeschichter Fettaufschwemmung gleichzeitig mit Insulin und Glukose weiter behandelt.

\begin{tabular}{|c|c|c|c|c|c|}
\hline $\begin{array}{l}\text { Versuchs- } \\
\text { tier-Nr. }\end{array}$ & $\begin{array}{c}\text { Insulin (E) } \\
\text { pro kg }\end{array}$ & $\begin{array}{l}\text { Gewicht } \\
\text { (g) }\end{array}$ & $\begin{array}{c}\text { Gewieht des } \\
\text { Herzens (g) }\end{array}$ & $\begin{array}{l}\text { Glykogen } \\
(\%)\end{array}$ & $\begin{array}{l}\text { Fett } \\
(\%)\end{array}$ \\
\hline 61 & 0,5 & 1800 & 3,8 & 0,488 & 2,51 \\
\hline 62 & 0,5 & 1850 & 4,1 & 0,512 & 2,72 \\
\hline 63 & 0,5 & 1800 & 4,0 & 0,518 & 2,84 \\
\hline \multicolumn{2}{|c|}{ Durehsehnitt } & & & 0,506 & 2,69 \\
\hline 64 & 1,0 & 1980 & 4,1 & 0,331 & 2,48 \\
\hline 65 & 1,0 & 2000 & 4,0 & 0,398 & 3,28 \\
\hline 66 & 1,0 & 2200 & 4,5 & 0,349 & 3,26 \\
\hline \multicolumn{2}{|c|}{ Durchschnitt } & & & 0,359 & 3,01 \\
\hline
\end{tabular}

Tabelle 18.

Derselbe Versuch wie oben, aber Lävulose statt Gluliose verwendet.

\begin{tabular}{|c|c|c|c|c|c|}
\hline $\begin{array}{l}\text { Versuchs- } \\
\text { tier-Nr. }\end{array}$ & $\begin{array}{c}\text { Insulin }(\mathbf{E}) \\
\text { pro kg }\end{array}$ & $\begin{array}{c}\text { Gewicht } \\
(\mathrm{g})\end{array}$ & $\begin{array}{l}\text { Gewicht des } \\
\text { Herzens (g) }\end{array}$ & $\begin{array}{c}\text { Glykogen } \\
(\%)\end{array}$ & $\begin{array}{l}\text { Fett } \\
(\%)\end{array}$ \\
\hline $\begin{array}{l}67 \\
68 \\
69\end{array}$ & $\begin{array}{l}0,5 \\
0,5 \\
0,5\end{array}$ & $\begin{array}{l}1900 \\
1690 \\
2100\end{array}$ & $\begin{array}{l}4,3 \\
3,8 \\
5,0\end{array}$ & $\begin{array}{l}0,487 \\
0,511 \\
0,445\end{array}$ & $\begin{array}{l}2,78 \\
2,70 \\
2,95\end{array}$ \\
\hline \multicolumn{2}{|c|}{ Durchsehnitt } & & & 0,481 & 2,81 \\
\hline $\begin{array}{l}70 \\
71 \\
72\end{array}$ & $\begin{array}{l}1,0 \\
1,0 \\
1,0\end{array}$ & $\begin{array}{l}2000 \\
2000 \\
1800\end{array}$ & $\begin{array}{l}4,8 \\
5,0 \\
4,1\end{array}$ & $\begin{array}{l}0,399 \\
0,285 \\
0,325\end{array}$ & $\begin{array}{l}3,24 \\
3,10 \\
\mathbf{2}, 99\end{array}$ \\
\hline \multicolumn{2}{|c|}{ Durehschnitt } & & & 0,336 & 3,11 \\
\hline
\end{tabular}

Dass die Fettablagerung im Herzmuskel durch Zuckergabe gewissermassen abgeschwächt wird, ist schon im vorangehenden Versuch (Tabelle 6 und 7) festgestellt. Im Vergleich mit den dabei gefundenen Zahlen erweist sich das Resultat der Insulinanwendung aus diesem Ver- 
such keineswegs als günstig für das Unterdrücken der Fettablagerung, indem der Glykogengehalt des Herzmuskels durch Zusammenwirken des Hormons eher vermindert wurde als ohne dies.

\section{Einfluss des Insulins auf den Fettgehalt des Herzmuskels der phosphorvergifteten Tiere.}

Es ergab sich aus dem letzten Versuch, dass zur Beseitigung des nach Infusion einer Fettaufschwemmung im Herzmuskel abgelagerten Fettes durch Zuckergabe der Zusatz von Insulin eher nachteilig wirkt.

Es fragt sich nun, wie sich der Insulinzusatz zur Zuckerbehandlung der durch Phosphorvergiftung verursachten Herzmuskelverfettung verhält. Es wurde schon aus Versuch II, 2 festgestellt, dass der Fettgehalt des vergifteten Herzmuskels durch Zuckerbehandlung erheblich vermindert wird. Im vorliegenden Versuch wurde sodann weiter untersucht, ob auf den vergifteten Herzmuskel die kombinierte Insulin-Zuckerapplikation besser wirkt als die einfache Zuckerbehandlung.

\section{Einfache Insulinbehandlung der Phosphortiere.}

Zunächst wurder die phosphorvergifteten Tiere mit Insulin allein behandelt.

48 Stunden nach Gabe von Phosphor auf dem eingangs erörterten Weg bekamen die Kaninchen $3 \mathrm{mal}$ hintereinander im Intervalle von 22,5 Stunden eine Menge Insulin von 0,5 bzw. 1,0 E. H. pro kg unter die Haut und 3 Stunden nach der letzten Injektion wurden sie getötet.

Tabelle 19.

Glykogen- und Fettgehalt des Herzmuskels der Phosphortiere nach Insulinbehandlung.

\begin{tabular}{|c|c|c|c|c|c|}
\hline $\begin{array}{l}\text { Versuchs- } \\
\text { tier-Nr. }\end{array}$ & $\begin{array}{c}\text { Insulin }(\mathbf{E}) \\
\text { pro kg }\end{array}$ & $\begin{array}{l}\text { Gewieht } \\
\text { (g) }\end{array}$ & $\begin{array}{l}\text { Gewicht des } \\
\text { Herzens (g) }\end{array}$ & $\begin{array}{c}\text { Glykogen } \\
(\% 6)\end{array}$ & $\begin{array}{l}\text { Fett } \\
(\%)\end{array}$ \\
\hline $\begin{array}{l}73 \\
74 \\
75\end{array}$ & $\begin{array}{l}0,5 \\
0,5 \\
0,5\end{array}$ & $\begin{array}{l}2400 \\
2600 \\
2300\end{array}$ & $\begin{array}{l}4,5 \\
5,3 \\
4,5\end{array}$ & $\begin{array}{l}0,248 \\
0,160 \\
0,176\end{array}$ & $\begin{array}{l}3,85 \\
3,66 \\
3,31\end{array}$ \\
\hline \multicolumn{2}{|c|}{ Durohsehnitt } & & & 0,195 & 3,60 \\
\hline $\begin{array}{l}76 \\
77 \\
78\end{array}$ & $\begin{array}{l}1,0 \\
1,0 \\
1,0\end{array}$ & $\begin{array}{l}2400 \\
2000 \\
2300\end{array}$ & $\begin{array}{l}5,8 \\
3,9 \\
5,0\end{array}$ & $\begin{array}{l}0,168 \\
0,224 \\
0,152\end{array}$ & $\begin{array}{l}5,75 \\
4,00 \\
5,40\end{array}$ \\
\hline \multicolumn{2}{|c|}{ Durchschnitt } & & $\therefore$ & 0,181 & 5,04 \\
\hline
\end{tabular}


Das Ergebnis (Tabelle 19) zeigt uns, dass das Insulin die Organverfettung infolge Vergiftung gar nicht unterdruickt, sondern den Fettgehalt des Herzmuskels dabei vergrössert, und zwar um so mehr, je mehr Insulin verabreicht wird, was schon Naga o in dieser Klinik bei Phosphorleber beobachtet hat.

\section{Kombinierte Insulin-Glukosebehandlung der Phosphortiere.}

In diesem eig'entlichen Versuche über den Erfolg der Insulin-Zukkertherapie wurde die Zufuhr von Insulin und Zucker genau so wie bei Versuch III, 1 und II, 2 ausgeführt.

Wie man aus Tabelle 20 ersieht, betrug der Glykogenwert bei Anwendung von 0,5 E. H. Insulin $0,63 \%$, bei 1,0 E. H. $0,48 \%$, und der des Fettes bei ersterem 3,84 und bei letzterem 3,89\% (Tabelle 20).

Vergleicht man diese Zahlen mit dem Ergebnis des Versuches II, 2 (Tabelle 11), so erkennt man, dass der Fettgehalt des Herzmuskels der Phosphortiere nach kombinierter Behandlung mit Insulin und Glukose eher höher ist als der nach einfacherZuckerbehandlung, wobei der Glykogengehalt das umgekehrte Verhältnis zeigt.

Tabelle 20.

Glykogen- und Fettgehalt des Herzmuskels der Phosphoritiere nach Tnsulin-Traubenzuckerbehandlung.

\begin{tabular}{|c|c|c|c|c|c|c|}
\hline $\begin{array}{l}\text { Versuchs- } \\
\text { tier-Nr. }\end{array}$ & $\begin{array}{c}\text { Insulin }(E) \\
\text { pro kg }\end{array}$ & $\begin{array}{c}\text { Glukose }(\mathrm{g}) \\
\text { pro kg }\end{array}$ & $\begin{array}{l}\text { Gewicht } \\
\text { (g) }\end{array}$ & $\begin{array}{l}\text { Gewicht des } \\
\text { Herzens }(g)\end{array}$ & $\begin{array}{c}\text { Glykogen } \\
(\%)\end{array}$ & $\begin{array}{l}\text { Fett } \\
(\%)\end{array}$ \\
\hline 79 & 0,5 & 2,5 & 2600 & 5,8 & 0,792 & 3,77 \\
\hline 80 & 0,5 & 2,5 & 2600 & 5,6 & 0,496 & 3,20 \\
\hline 81 & 0,5 & 2,5 & 2600 & 6,2 & 0,596 & 4,49 \\
\hline \multicolumn{2}{|c|}{ Durchsehnitt } & & & & 0,628 & 3,84 \\
\hline 82 & 1,0 & 2,5 & 2050 & 4,7 & 0,526 & 3,79 \\
\hline 83 & 1,0 & 2,5 & 2000 & 5,5 & 0,424 & 3,96 \\
\hline 84 & 1,0 & 2,5 & 1900 & 4,2 & 0,496 & 3,92 \\
\hline \multicolumn{2}{|c|}{ Durehsehnitt } & & & & 0,482 & 3,89 \\
\hline
\end{tabular}

\section{Kombinierte Insulin-Lävulosebehandlung der Phosphortiere.}

Aus einem sonst genau wie oben, aber mit Lävulose angestellten Versuche ergab das Herzglykogen bei $0,5 \mathrm{E}$. H. Insulin $0,68 \%$, bei 1,0 E. H. 0,50\% und dementsprechend das Fett 3,41 bzw. 3,71\% (Ta- 
belle 21). Auch in diesem Lävuloseversuch erwies sich der Glykogenbestand des Herzmuskels der Phosphortiere bei gleichzeitiger Anwendung des Insulins als unguinstiger als bei einfacher Zuckerapplikation. Bei Zuckerbehandlung der mit Herzmuskelverfettung einhergehenden Krankheitszustände ist also der Insulinzusatz eher nachteilig.

Tabelle 21.

Derselbe Versuch wie oben, aber Lävulose statt Glukose verwendet.

\begin{tabular}{|c|c|c|c|c|c|c|}
\hline $\begin{array}{l}\text { Versuchs- } \\
\text { tier-Nr. }\end{array}$ & $\begin{array}{c}\text { Insulin (E) } \\
\text { pro } \mathrm{kg}\end{array}$ & $\begin{array}{c}\text { Lärulose }(g) \\
\text { pro } \mathrm{kg}\end{array}$ & $\begin{array}{c}\text { Gewicht } \\
\text { (g) }\end{array}$ & $\begin{array}{l}\text { Gewicht des } \\
\text { Herzens (g) }\end{array}$ & $\begin{array}{c}\text { Glykogen } \\
(\%)\end{array}$ & $\begin{array}{l}\text { Fett } \\
(\%)\end{array}$ \\
\hline 85 & 0,5 & 2,5 & 2450 & 4,9 & 0,632 & 3,81 \\
\hline 86 & 0,5 & 2,5 & 1970 & 4,5 & 0,588 & 3,90 \\
\hline 87 & 0,5 & 2,5 & 2230 & 5,1 & 0,822 & 3,54 \\
\hline \multicolumn{2}{|c|}{ Durchschnitt } & . & & & 0,681 & 3,41 \\
\hline 88 & 1,0 & 2,5 & 2410 & 5,5 & 0,473 & 3,27 \\
\hline 89 & 1,0 & 2,5 & 2520 & 6,0 & 0,498 & 3,59 \\
\hline 90 & 1,0 & 2,5 & 1830 & 4,3 & 0,535 & 2,73 \\
\hline \multicolumn{2}{|c|}{ Durchschnitt } & & & & 0,502 & 3,71 \\
\hline
\end{tabular}

\section{Besprechung der Ergebaisse.}

Der vorliegende Versuch wurde unternommen, um zu erfahren, ob die neuerdings von manchen Autoren so warm empfohlene InsulinZuckerbehandlung gegen verschiedene Herzkrankheiten im Sinne der Glykogenbereicherung im Herzmuskel zurecht besteht oder nicht. Die Untersuchungen ergaben, dass eine gegenseitige Verdrängung des Glykogens und Fettes, die schon von manchen Autoren im Leberparenchym bestätigtt wurde, auch im Herzmuskel besteht und dies sowohl für das normale als auch für das phosphorvergiftete Horz gilt. Dadurch gewinnt die Anwendung der Zuckerinfusion zum Zwecke der Hebung der Herzkraft und der Beseitigung der Verfettung eine rationelle Stütze.

Besonders beim erkrankten Herzen ist es empfehlenswert, zu diesem. Zwecke Lävulose der Glukose vorzuziehen, da man sich durch erstere einen besseren Erfolg versprechen kann. Die Vorzüglichkeit der Lävulose als Glykogenbildner, die sich bei erkrankter Leber bereits erwiesen hat, wurde in diesem Versuch auch beim phosphorvergifteten Herzmuskel bestätigt.

Diese fettvertilgende und glykogenbereichernde Wirkung der Zuckerinfusion wurde durch Insulinzusatz gar nicht gefördert, sondern 
im Gegenteil mehr oder minder stark beeinträchtigt. Diese schädliche Wirkung nimmt mit der Menge des angewendeten Hormons immer zu. Wie O tomo in dieser Klinik bei Frosch- und Kaninchenherzen experimentell feststellte, muss deswegen der Insulinzusatz bei dor Zuckerbehandlung der Herzkrankheiten ausnahmslos als überflüssig betrachtet werden.

\section{Zusammenfassung.}

1. Durch intravenöse Zuckerinfusion kann man einen Glykogenansatz im Herzmuskel und zugleich eine Fettvertilgung erzielen und durch intravenöse Fetteinverleibung gerade das Umgekehrte. Es besteht somit auch im Herzmuskel gegenseitige Verdrängung des Glykogens und Fettes wie in der Leber.

2. Die bei Phosphorvergiftung zustande kommende Fettansammlung im Herzmuskel wird durch aus infundiertem Zucker entstandenen Glykogenansatz in erheblichem Masse unterdrückt. Dabei ist Lärulose als Fettvertilger ebenso wie auch als Glykogenbildner etwas wirksamer als Glukose, was auch bei Phosphorleber bestätigt wird.

3. Insulinzusatz erweist sich gar nicht als vorteilhaft bei der Zuckerbehandlung des erkrankten Herzens. Der Glykogenansatz wird dadurch nicht nur nicht gefördert, sondern stets mehr oder minder stark verhindert.

\section{Literatur.}

(1) B üdinge n, Ernährungsstörungen des Herzmuskels, Leipzig 1917.

(2) Külbs, Mohr u. Sta ehelins Handbuch der inneren Medizin, Berlin 1928, 2,4 .

(3) Lewis, Ebenda.

(4) Lasch, Z. ges. exp. Med., 1932, 83, 36.

(5) Is him ori, Chuo Igakkai Zasshi, 1914, 117, 21.

(6) Valdes, Arch. f. path. Anat. n. Physiol., 1930, 274, 361.

(7) Costa, Zit. nach Kongr. Zbl. ges. inn. Med., 1930, 57, 456.

(8) Pomod oro, Zit. nach Kongr. Zbl. ges. inn. Med., 1933, 72, 272.

(9) Osato, Z. ges. exp. Med., 1926, 51, 488.

(10) Loeper, Lema ire u. Degos, Presse Méd., 1930, 38, 1361.

(11) Kisthiniosu. Gomez, Presse Med., 1930, 38, 1363.

(12) Rimbaud, Balmè s, Anselme-Marti n, Presse Méd., 1931, 39, 1647.

(13) Machi oro, Riforma Med., 1931, 2, 1297.

(14) Bagnaresi, Rass. Ter. e Pat. Clin., 1931, 3, 475.

(15) Bobbio u. Mas sobrio, Minerva Med., 1931, 22, 637.

(16) Penati, Baroneu. Croce, Minerva Med., 1931, 22, 649.

(17) Ginkel, Med. Kl., 1932, 1724. 
(18) Schäffer, Buckan. Friedländer, Z. ges. exp. Med., 1927, 57, 35.

(19) Eismayer u. Quincke, Zschr. Biol., 1930, 90, 57.

(20) Ernstene u. Altschule, J. clin. Invest., 1981, 10, 521.

(21) Morawitz, Dtsch. med. W., 1935, 45.

(22) Ot o m o, Tohoku Igaku Zasshi, 1936, 19, 690.

(23) Mo Cormik u. Ma cle od, Transact. roy. Soc. Canada, 1923, 17, 63.

(24) Michelazzi, Pathologica (Genova), 1932, 24, 293.

(25) Hepburn u. Latehford, Am. J. Physiol, 1922, 62, 177.

(26) Babkin, Brit. J. Exp. Path., 1423, 4, 310,

(27) Ochiai, J. Oriental. Med., 1927, 7, 589.

(28) Arndt, Beitr. z. path. Anat. n. z. allg. Path., 1928, 79, 69 u. 523.

(29) L aw rence u. Mc Cance, Biochem. J., 1931, 25, 570.

(30) Edwards u. Page, Am. J. Physiol., 1924, 69, 177.

(31) N aga s u e, Tokio Igakwai Zasshi, 1926, 40, 1 u. 25.

(32) Cruickshank u. Shrivastava, Am. J. Physiol., 1930, 92, 144.

(33) No m u ra, Tohoku J. Exp. Med., 1929, 121, 247.

(34) Tak a hat a u. Ku mo, Fukuoka Ikadaigaku Zasshi, 1926, 19, 169 .

(35) Ku magawa u. Suto, Biochem. Z. 1908, 8, 212.

(36) Hagedorn u. Jensen, Biochem. Z., 1923, 135, 46.

(37) Wegeli n, Berlin. kl. W., 1913, 2125 u. 2190.

(38) Mlod ows k a, Beitr. z. path. Anat. u. z. allg. Path., 1918, 64, 18.

(39) Junkersdorf r. Kü h n, Arch. f. d. ges. Physiol., 1926, 208, 617.

(40) Gü r ber, Sitzungsber. d. Physical. Med. Ges. zu. Würzburg, 1895. Zit. naeh Berblinger, Beitr. z. path. Anat. u. 3, allg. Path., 1912, 53, 155.

(41) Sato, Tohoku J. Exp. Med., 1923, 4, 312.

(42) Nagao u. Otom o, Nippon Naikwagakkwai Zasshi, 1934, 22, 1116.

(43) Naga o, Tohoku J. Exp. Med., 1934, 24, 529.

(44) Rosenfeld, Berlin. kl. W., 1904, 587.

(45) Klewitz u. Kirchheim, kl. W., 1922, 1397.

(46) Sakamaki, Tokio Iji Shinshi, 1936, 2976. 